the GDPs ensure that the oral cavity is in good health prior to planned cardiac surgery. It may therefore be more appropriate that similar forms are signed at the completion of dental treatment by their GDP. The above may not apply to emergency cardiac surgery when patients are unable to attend their dentist.

It is understandable that in certain cases the decision to declare dental fitness beyond any doubt can be difficult and overwhelming for the dentist. The lack of clear guidance, the patient's compliance with everyday oral care and the shift to maintaining the natural dentition is contributing further to the uncertainty.

A. Pantiora, by email

1. Chambers J B, Dayer M, Prendergast B D et al. British Heart Valve Society Beyond the antibiotic prophylaxis of infective endocarditis: the problem of dental surveillance. Heart 2013; 99: 363-364.

DOI: $10.1038 /$ sj.bdj.2017.382

\section{Water fluoridation}

\section{It really is this simple}

Sir, thank you for printing Dr Connett's letter regarding fluoride. ${ }^{1}$

Recent publicity around unacceptable levels of child GA exodontia and the development of the (welcomed) Starting Well Programme, targeting prevention at the 13 areas in England with the worst child dental health, make the letter highly relevant.

There is simply no credible evidence that fluoride is neurotoxic at the concentrations used in community water fluoridation or those occurring naturally in areas like Hartlepool of between 0.7 and $1 \mathrm{ppm}$. There is, however, an international consensus that fluoridation at this level is safe and effective. It really is this simple.

The language used by Dr Connett is typical of the anti-fluoridation lobby. Words such as neurotoxic, toxic, fearful, chemophobia are designed to elicit an emotional response and to intimidate - even when there is zero evidence to support the points made. To an extent this approach has worked, successfully intimidating dentists and politicians even in the face of the scientific evidence that fluoridation can make a difference and is safe. There are signs that this is changing with the realisation that, in spite of the improved evidence-based prevention being delivered by GDPs in primary care, problems remain and fluoridation could help to change this.

It really is time that science and evidence is allowed to make the case for and against fluoridation. Recent reports ${ }^{2-4}$ from New Zealand, Australia and here in the UK state resoundingly that fluoridation is safe and reduces dental disease. As dentists, we need to demonstrate leadership, talk to our patients and local politicians and advocate fluoridation, particularly in communities where the dental health burden is high and where dental health inequalities are wide. It is time that the facts around fluoridation are allowed to speak for themselves - to use Dr Connett's own words - to protect the health of citizens.

Hull and the East Riding of Yorkshire LDC

1. Connett P. Water fluoridation: Is fluoride chemophobia? Br Dent J 2017; 222: 323-324.

2. The Royal Society of New Zealand. Health effects of water fluoridation: A review of the scientific evidence. A report on behalf of the Royal Society of New Zealand and the Office of the Prime Minister's Chief Science Advisor. August 2014.
Available at: http://royalsociety.org.nz/assets/documents/ Health-effects-of-water-fluoridation-Aug-2014-corrected-Jan-2015.pdf (accessed April 2017).

3. NHMRC. Draft Information Paper: Effects of water fluoridation on dental and other outcomes. September 2016. Available at: https://consultations.nhmrc.gov.au/ files/consultations/drafts/nhmrc-fluoride-information.pdf (accessed April 2017).

4. Public Health England. Water fluoridation. Health monitoring report for England 2014. Executive summary. March 2014. Available at: https://www.gov.uk/ government/uploads/system/uploads/attachment_data/ file/300201/Water_fluoridation_health_monitor_for_ England_2014_executive_summary_1Apr2014.pdf (accessed April 2017).

DOI: $10.1038 /$ sj.bdj.2017.383

\section{Mystery object}

\section{Denture drop}

Sir, I acquired but sadly disposed of an identical 'mystery object' to that pictured in the $B D{ }^{1}$ (Fig. 1) when I took over a retirement practice in Woodford Green in 1978. It was designed to be held by the dentist or nurse, just above the chin, allowing discreet removal of dentures directly into the small bowl. Patients would use their hands to briefly 'hide' the dentures as they slipped out of the mouth.

C. Wallis, by email

1. News. Can you identify this mystery object? Br Dent J 2017; 222: 325

DOI: 10.1038/sj.bdj.2017.384

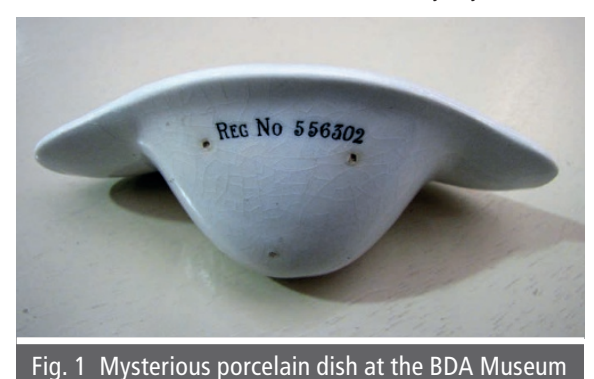

\title{
CASE REPORT LETTERS
}

\section{TMJ pain}

\section{Giant cell arteritis warning}

Sir, in August of 2016 a very fit and healthy 73-year-old female patient of mine developed intermittent severe pains in the occipital area bilaterally. Following a series of tests, a consultant rheumatologist prescribed prednisalone $25 \mathrm{mg}$ per day but admitted to not having a definitive diagnosis.

In December 2016 the patient suddenly developed severe TMJ pain and although no occlusal disharmony in two intact arches was identified a soft-bite raising splint was provided. This gave some slight relief and the symptoms slowly subsided. The prednisalone was then at $10 \mathrm{mg}$ per day.

In January 2017 she noticed that blood vessels in her temple were becoming prominent (but not painful) and then she experienced pain in her muscles of mastication on chewing (claudication) and was unable to finish a meal where meat required chewing.

An urgent appointment with the rheumatologist was sought. He diagnosed giant cell arteritis (GCA) and the prednisalone was increased to $60 \mathrm{mg}$. A significant number of patients with GCA (up to $20 \%$ ) suffer problems with occlusion of the opthalmic artery and permanent blindness. Strokes are also more likely. It is therefore essential that high doses of prednisalone are given as soon as GCA is suspected as this massively decreases the incidence of eye problems.

As general dental practitioners we should be more aware of GCA and that pain on eating or other undiagnosed 'jaw pain', is a symptom. Also, an oral surgeon advised me that any instance of rapid onset TMJ pain in an older patient should be referred urgently and that a biopsy of the temporal artery would probably be carried out. It seems there 
may be a connection between patients with polymyalgia rheumatica (PMR) and GCA

4 and a significant proportion of patients with PMR also get GCA. It may be that arteritis in blood vessels supplying muscles results in a diminished supply thereby giving rise to the symptoms of PMR. Where patients report a diagnosis of polymyalgia I will now ask them if there are any headaches or jaw pain, especially on eating.

T. Lynn, by email

DOI: $10.1038 /$ sj.bdj.2017.385

\section{OMFS}

\section{Odontalgia and facial pain}

Sir, an 83-year-old gentleman recently attended our OMFS department with intractable facial pain of presumed dental origin. He initially reported a 6-month history of gradually worsening pain in the upper left quadrant. This became increasingly severe and he eventually visited his dentist who assumed a dental causes and extracted 25, 26 and 27 (Fig 1.)

However, following these extractions, the pain became increasingly relentless and he attended the accident and emergency department with referral to OMFS. His pain was now severe left sided pain radiating from the upper lip to the left ear. The pain was scored $10 / 10$ and described as a sharp, electric shock like, episodic pain with a trigger point around 23, 24 region. An MRI scan was ordered which demonstrated a solid, space occupying lesion in the left cerebellopontine angle (CPA). This was reported to be causing

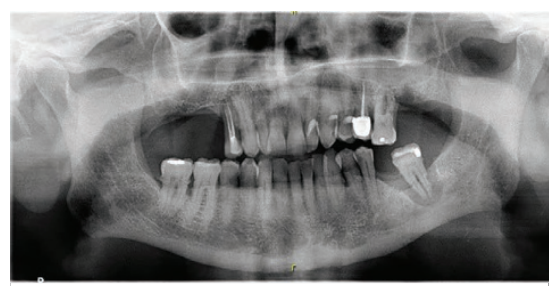

Fig. 1 An OPT showing 25 heavily filled, 26 distal radiolucent lesion and 37 apical radiolucent lesion

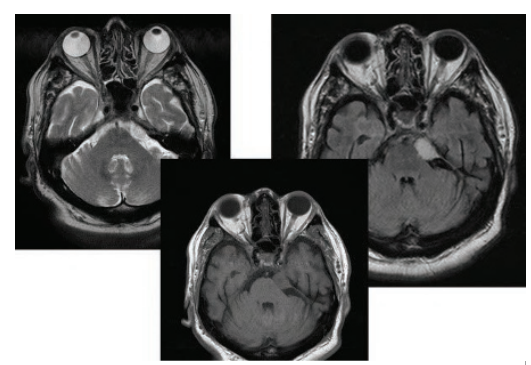

Fig. 2 Axial MRI images showing lesion in left CPA distortion on the left trigeminal nerve. The definitive diagnosis was a meningioma ${ }^{1}$ which was impinging on the left $\mathrm{V} 2$ trigeminal nerve resulting in neuralgia. ${ }^{2}$ Initial management was medical treatment with carbamazepine and a neurosurgical consult was also arranged.

This case serves to highlight the importance of a thorough history and clinical examination in all cases of facial pain, together with reassessment of those patients in whom clinical symptoms do not resolve following initial treatment. In these cases, a prompt referral to the appropriate specialist (in this case OMFS) is advised for further investigations and management.

\section{Davies, C. J. Mannion, by email}

1. Springborg J B, Poulsgaard L, Thomsen J. Nonvestibular schwannoma tumours in the Cerebellopontine angle: $\mathrm{A}$ structured approach and management guidelines. Skull Base 2008; 18: 217-227.

2. Zakrzewska J M, Linskey M E. Trigeminal neuralgia. BMJ 2014; 348: g474.

DOI: $10.1038 /$ sj.bdj.2017.386

\section{Osteonecrosis as a complication of GA}

Sir, we wish to present an interesting case of osteonecrosis of the jaw.

A 41-year-old female underwent general anaesthesia for hysterectomy. She had no history of treatment with bisphosphonates or radiation to the head and neck region. After recovery from general anesthesia, she started to feel pain from her right mandible. Twentytwo days after the general anaesthesia she went to her dentist for examination due to persisting pain. Examination revealed a mucosal ulcer and local bone exposure at the right mylohyoid ridge close to a mandibular tori. The patient was referred to an oral surgeon due to suspected oral malignancy. Seventeen days later (39 days after anaesthesia) the patient had a spontaneous exfoliation of a bone sequestrum in her mouth, and her symptoms declined almost immediately. At the time of examination by the oral surgeon (three days after exfoliation of the sequestrum), the ulcer was healing (Fig. 1). A CT scan showed signs of an exfoliated bone sequestrum (Fig. 2). Complete healing and absence of symptoms were confirmed 50 days after anaesthesia.

To our knowledge, only six cases with the same complication of general anaesthesia as described here have previously been reported, and never in a dentistry journal. ${ }^{1-2}$ We believe that this type of injury could either be caused by soft tissue necrosis due to local pressure from the endotracheal tube, or by soft tissue injury caused by the angulation and insertion of the laryngoscope, which could explain why patients are always affected on the lingual side of the right mandible. ${ }^{2}$ The blade of the laryngoscope is sharp and usually held in the left hand of the operator while inserted into the oral cavity along the right side of the mandible. In almost all reported cases, symptoms emerge directly after recovery from general anaesthesia or within a day.

Patients with large mandibular tori are at particular risk. Careful oral manipulation and use of techniques to facilitate laryngoscopy could possibly reduce the risk of oral trauma. ${ }^{3}$ Patients affected by this complication need to be followed until healing is confirmed (within 1-8 weeks). In selected cases, minimal surgical intervention may be needed to remove sequestered bone, to provide pain relief and to reduce the time required for healing.

M. Kharazmi, U. Nilsson, Västerås, Sweden P. Hallberg, Uppsala, Sweden

1. Almazrooa SA, Chen K, Nascimben L, Woo S B, Treister N. Case report: osteonecrosis of the mandible after laryngoscopy and endotracheal tube placement. Anesth Analg 2010; 111: 437-441.

2. Kharazmi M, Björnstad L, Hallberg P et al. Mandibular bone exposure and osteonecrosis as a complication of general anaesthesia. Ups J Med SCi 2015; 120: 215-216

3. Kharazmi M, Scheer $\mathrm{H}$, Hallberg P. Reduced obstacles, maximized vision (ROMV): a new technique to facilitate laryngoscopy for endotracheal intubation. Ups J Med Sci 2016; 16: 1-2.

DOI: $10.1038 /$ sj.bdj.2017.387

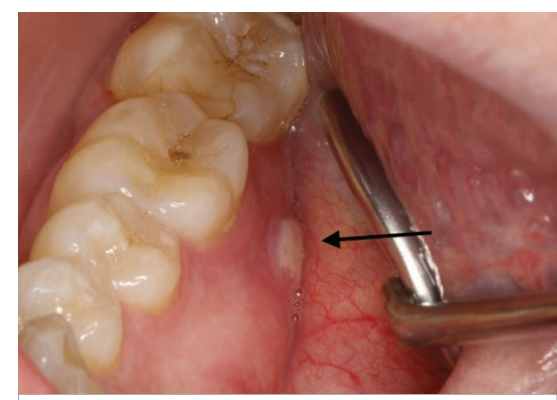

Fig. 1 A healing ulcer located at lingual shelf of the right mandible, on the posterior aspect of a mandibular tori

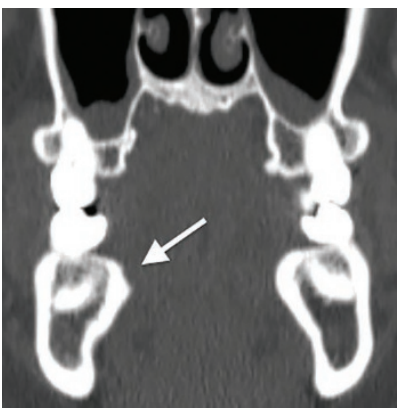

Fig. 2 CT scan showing reduced amount of bone after spontaneous exfoliation of a sequestrum on the lingual shelf of the right mandible 